\title{
TEMPERATURE DEPENDENCE OF SHEAR ELASTICITY OF SOME LIQUIDS
}

\author{
Ochir R.Budaevl, Bair B.Damdinovl, P. Adiyasuren, A.Tsend-Ayush ${ }^{1}$, \\ 1. Buryat Scientific Center of RAS (Sib. Div.), Ulan-Ude, Russia \\ 2. National university of Mongolia \\ p_adiyasuren@yahoo.com
}

\begin{abstract}
The temperature dependencies of real and imaginary parts of complex shear modulus of liquids have been obtained. It has shown that both real shear modulus G' and ft imaginary shear modulus G" decrease with increasing temperature. The experiments havebeen carried out by resonance method at a shear oscillation frequency of $74 \mathrm{kHz}$. Liquids of viscosity $0.02-0.03 \mathrm{~Pa}$-s have been investigated.
\end{abstract}

\section{Introduction}

A.V.Bulgadaev [1-3] who was 90 years old last year, began to research $>/$ mechanical properties of liquid with piezoequartz vibrator application in Ulan-Ude. A constant supervisor of works in Ulan-Ude for a long time was the academic B.V.Deryagin. Bulgadaev observed an increase in the resonance frequency of piezoelectric quartz $\left(/^{\circ}=10^{5} \mathrm{~Hz}\right)$, when the thin interlayer of a liquid is located between piezoelectric quartz and a second solid body (cover-plate) [15]. The interlayer of liquid test shear elasticity and assumed that it is the special mechanical properties of the boundary layers that appear under the action of solid body forces. If the liquid interlayer possesses shear elasticity the resonance frequency could only increase. Bazaron [4-6] has detected a hyperbolic dependence

Of resonance frequency shift $\mathrm{A} /$ ', , on thickness $\mathrm{H}$ of the investigated interlayer: $\mathrm{A} /{ }^{\prime} \mathrm{H}=$ const., where he used an interference method of liquid interlayer thickness measurement. It has been shown that the shear elasticity modulus G' of the liquid is not dependent on the thickness of film $\mathrm{H}$, when mass $\mathrm{M}$ and resonance frequency $f^{\circ}$, of piezoelectric quartz and also squares of cover-plate $\mathrm{S}$ a interlayer - cover - plate surface contact. Non - linear dependence $\mathrm{A} /$ ' on $1 / \mathrm{H}$ at different amplitudes of piezoelectric quartz oscillation has been obtained. Bazaron substitute the given piezoelectric quartz by the piezoelectric quartz of X-18.5 cut with zero Poisson's coefficient, and detected a linear dependence of A/' on 1/H. it has been shown that the shear elasticity of the liquid interlayer is not dependent on the field 


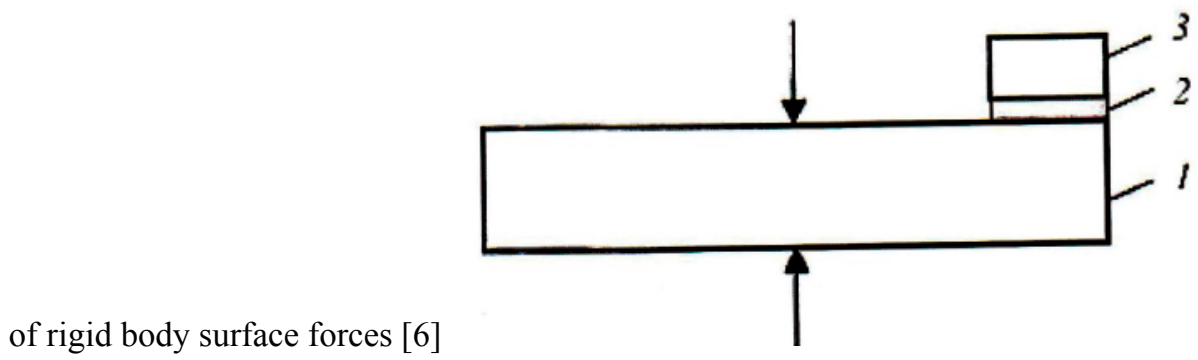

Fig 1 Piezoelectric quartz crystal 1 with a liquid layer 2 and a quirtz strap 3

One of the authors of this paper (O.R.Budaev) developed a theoretical method to measure the shear elasticity of a liquid at different intervals of thickness [7]. The appropriate calculated formulas are presented in Ref. [8]. The measurements of shear modulus at three different intervals of thickness [7]. The appropriate calculated formulas are presented in Ref. [8]. The measurements of shear modulus at three different intervals of thickness agreed satisfactorily with each other [9-11]. It has been shown that Iiquids possess a measurable shear modulus at $10^{5} \mathrm{~Hz}$. According to Frenkel's theory [12], shear elasticity could be detected at frequencies of 5-6 orders or over. It has been suggested that that 'there is an unknown earls low frequency viscoelastc relaxation process concerned with collective interactions. The collective effects should depend on a positional relationship and interactions of groups which includes many molecules . A relaxation time can on many orders exceed the time of settled existence of one molecule ... Detection of a low frequency viscoelastic relaxation plays an important role in understanding liquid matter' [13]. Shear elasticity is now detected in liquids at lower frequencies [14-16

\section{Results and discussion}

The authors of this paper present the following presumable mechanism of shear elasticity. Collective interactions in simple polymeric systems are divided on an internal operation between links of one molecules, and on external one between different molecules. Any isolated polymeric molecule has a finite number of Fig 3. 
O.R.Budaev et al /Advances in Colloid and Interface Science 104(2003 )307-310 309 Af', $\mathrm{Hz}$

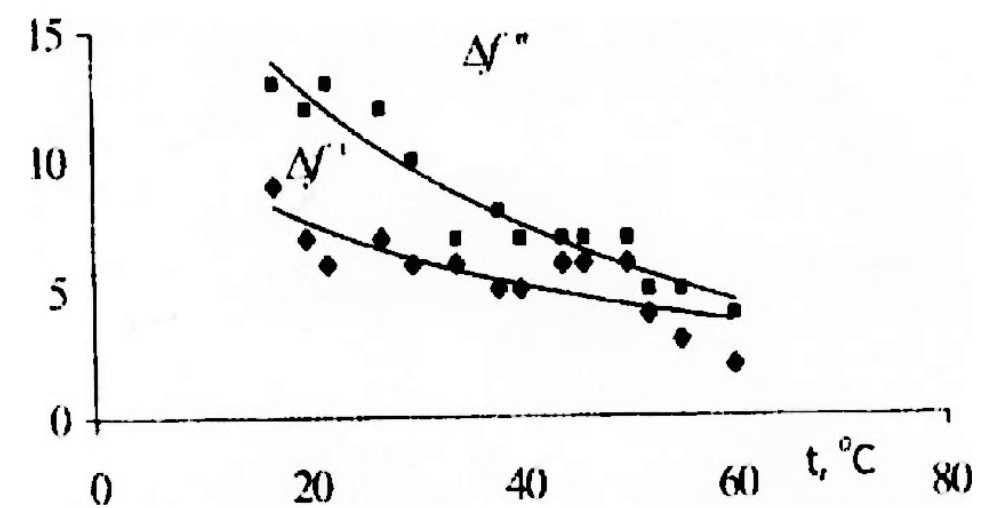

Fig. 2. Temperature dependence of real Af and imaginary Af" frequency shifts for polymethylsiloxane-100.

equilibrium space configurations. Each such configuration corresponds to a minimum of potential energy. The number of such configurations depends on the length of the polymeric molecule. Each molecule tends to be minimized in a tangle and the tangles tend to be dissolved in each other. Each tangle can consist of more than one molecule. The thermodynamic force of tangle arise. The dynamic equilibrium between these forces is installed. The un dissolved part of the tangle is termed a cluster, and the dissolved part of all tangles as a surface stratum of all clusters. Both the cluster size and thickness of the surface stratum are defined by temperature. The absence of clusters means a maximum of internal dissolution and for such a liquid the model of 'felt' by Ferry is usable [17]. We have a model of 'soft spheres' at the other extreme case. The polymeric chains ( $\mathrm{L}$ is its length) in the surface stratum are oriented along the thickness of this stratum and are in the surface stratum are oriented along the thickness of this stratum and are in the stretched ;state under the action of thermodynamic forces. Internal dissolution of polymeric molecules means formation of intermolecular forces between atoms of different molecules. The number of such links is equal $\mathrm{N}=\mathrm{pL}$, where $\mathrm{p}$ is the linear density of links. $\mathrm{N}$ links can be torn or stay at expansion. All links between molecules of different stratums can be broken under the action of small and fixed shear effort and the liquid will begin to flow. The pattern varies under a sine-wave shear effort. All links break due to step-by-step accumulation of energy. We suppose that the duration of this transition period is greater than the relaxation period of a single link. The relaxation period of a single link is evaluated by a single link. The relaxation period of a single links is evaluated by a magnitude of approximately 10 "9 $\mathrm{s}$ [15]. When length L is sufficiently great, all links have not enough time to be torn within a halt - period of an oscillation. The average number of links saved for one period of oscillation determines the shear elasticity of the liquid. The suggested mechanism shows the defining role of surface stratum, its width, and density of links be- 
tween molecules of different clusters inside this stratum on the viscoelastic behavior of a liquid under periodic action.

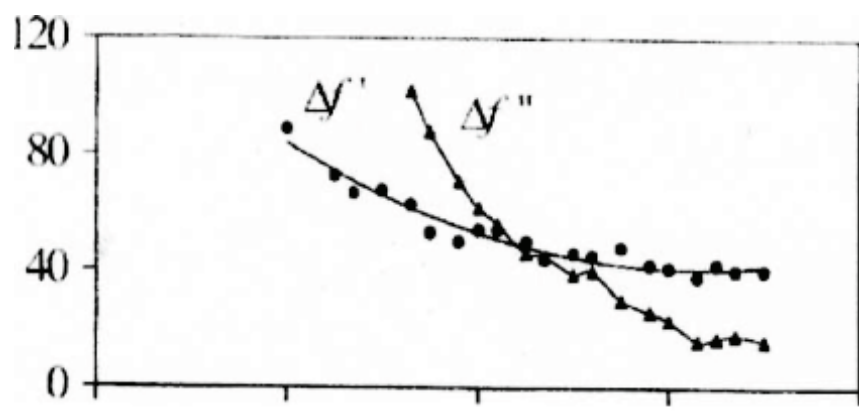

\section{$0 \quad 20 \quad 40 \quad 60^{\mathrm{r}-}{ }^{\circ} \quad 80$}

Fig. 3. Temperature dependence of real $A f$ and imaginary $A f$ " frequency shifts lor oleic acid.

show the temperature dependence of real $\boldsymbol{A} \boldsymbol{f}$ and imaginary $\boldsymbol{A} \boldsymbol{f}$ " frequency shifts of quartz resonator for PMS-100 and oleic acid correspondingly. It is visible that $\boldsymbol{A} \boldsymbol{f}$ and $\boldsymbol{A f}$ ", and real G' and imaginary G' shear module decrease with increasing temperature. The result is decreasing average length with increasing temperature. This decreasing may be explained for two reasons. At first, the activation energy of single links with growth of temperature is diminished. Secondly, the fluctuation free volume is augmented [18], i.e. movement of links of one tangle inside another are diminished. Such a feature is also observed more so in short molecules than in polymeric • types. In this case, non-dissolved parts of molecules, which can be presented as clusters, are diminished and even fade. The thermodynamic forces of inter dissolution operate already from both sides. Such a transition is possible for liquids with simple molecules such as water. In this case, their electrons take part in 'inter dissolution'. The relaxation frequency of simple liquids become mush higher than those of experiments. Investigations in the regard are ongoing . 
References

1. A.V.Bulgadaev,Pap. Buryat State Pedagog.Inst.(Russ) 8(1955) 27

2. A.V.Bulgadaev,Pap.BSPI .(Russ) 10 (1956) 41

3. A.V. Bulgadaev,Pap.BSPI .(Russ) 15 (1958) 12

4. U.B. Bazaron, B.V. Dejaguin, A.V, Bulgadaev, Dokl. AN SSSR 160 (1965) 799

5. U.B. Bazaron, B.V. Dejaguin, A.V, Bulgadaev, Dokl. AN SSSR 166 (1966) 639

6. U.B. Bazaron, B.V. Dejaguin, A.V, Bulgadaev, Sov.Phys.(JETP)51 (1966) 969

7. U.B. Bazaron, B.V. Dejaguin, A.V, Bulgadaev, Dokl. AN SSSR 205 (1972) 1326

8. K.T.Zandanova, B.V. Dejaguin, U.B. Bazaron, O.R. Budaev, Dokl. AN SSSR 215 (1974) 309

9. B.B. Bazaron, B.V. Dejaguin, O.R. Budaev B.B. Badmaev, , Dokl. AN SSSR 238 (1978) 50

10. B.B. Badmaev, B.B. Bazaron, O.R. Budaev, K.T.Zandanova, B.V. Dejaguin, Dokl. AN SSSR 254 (1980) 381

11. B.B. K.T.Zandanova, d Badmaev, B.B. Bazaron, O.R. Budaev, L.B.Dandaron, B.V. Dejaguin, K.T.Zandanova, et al ., Colloid.J.(Russ) 54 (1982) 841

12. Ya.1. Frenkel, Kinetic Theory of Liquid, Nauka . Leningrad, 1975

13. U.B. Bazaron, Low Frequency Shear Elasticity of RAS, Ulan-Ude, 2000

14. S.A. Bal'ginov, B.B.Badmaev, E.R.Ochirova, Acoust, Phys. 47 (2001) 785

15. B.B.Badmaev B.B. Damdinov, Acoust. , Phys. 47 (2001) 487

16. U.B. Bazaron, J. Phys. Chem.(Russ) 67 (1993) 1752

17. G.V. Kozlov, V.U. Novikov, Sov. Phys. 171(2001) 717

18. D.S. Sanditov, G.M. Bartenev, Physical Properties of Unregulated Structures, Nauka, Novosibirsk, 1982 